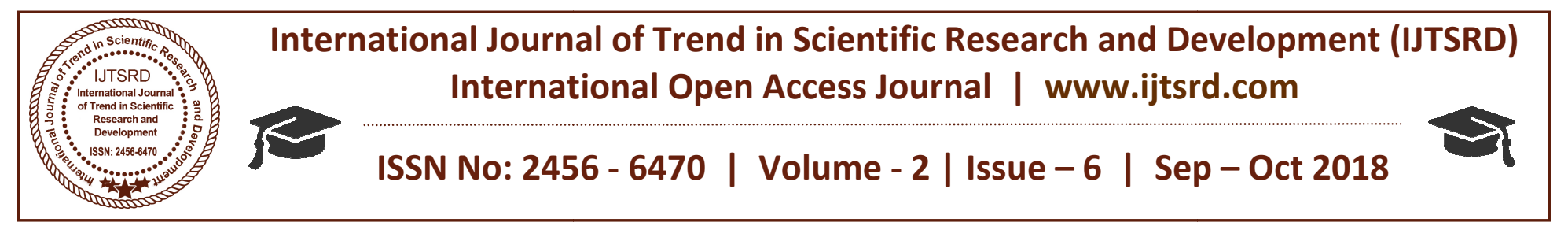

\title{
Wireless Linked Navel Substation for International Boundary Scanning and Surveillance System Using GPS
}

\author{
R. Mahalakshmi \\ Leturer, Department of Computer Engineering, \\ NPA Centenary Polytechnic College, Kotagiri, Tamil Nadu, India
}

\begin{abstract}
The boaters may sometimes cross their area limit without their knowledge. This causes a lot of problems. They may be caught by the other peoples. This project is developed for the boat users to find out their border in the sea area. The main modules in this project are RF transducer, microcontroller unit and LCD display. The Zigbee transmitter is connected at the border area. It transmits RF signals within the particular limit. The Zigbee receiver with the micro controller unit is connected at the boat. When the boat reaches the particular area, the RF signals are received by the receiver and given to the micro controller unit. The micro controller analyses the signal and calculates the distance and sends corresponding message to the LCD display. If it crosses the limit, the micro controller operates the warning buzzer and it switches off the running motor of the boat. Thus the boat may be automatically off and the boater may easily understand the situation. The micro controller program is written in embedded c language and the microcontroller used is ARDUINO.
\end{abstract}

Keyword: Air pollution, wireless sensor system, carbon monoxide, smoke, PHP-MYSQL, Lab VIEW

\section{INTRODUCTION}

There Have Been Several Alleged Incidents of Firing on Indian Fishermen Fishing In Palk Bay ${ }^{\text {Indian }}$ Government Has Always Taken Up The Issue of Safety Of Indian Fishermen On A Priority Basis With The Government Of Sri Lanka. Presently There Is No Bona fide Indian Fisherman in the Sri Lankan Custody. A Joint Working Group (JWG) Has Been Constituted To Deal With The Issues Related To Indian Fishermen Straying In Sri Lankan Territorial Waters, Work Out Modalities For Prevention Of Use Of Force Against Them And The Early Release Of
Confiscated Boats And Explore Possibilities Of Working Towards Bilateral Arrangements For Licensed Fishing. The JWG Last Met In Jan 2006. India Officially Protested Against Sri Lanka Navy For Its Alleged Involvement In Attacks On Indian Fishermen On January 12, 2011. Even After The Official Protest, Another Fisherman Was Killed In A Brutal Manner On Jan 22, 2011. Over 530 Fishermen Have Been Killed In The Last 30 Years. The Apathetic Attitude Of The Indian Government And The National Media Towards The Alleged Killing Of Tamil Nadu Fishermen By The Sri Lankan Navy Is Being Strongly Condemned.

\section{2IIPROPOSED SYSTEM}

It Consists Of Client Side Module Which Is Placed On The Boat And Server Side Module. Client Module Consists Of Microcontroller Unit Connected To The GPS Transceiver And Indication Unit Of LED And Buzzer. Server Module Consists Of System Control Unit And GPS Transceiver.

Zigbee Is A Specification For A Suite Of High Level Communication Protocols Used To Create Personal Area Networks Built From Small, Low-Power Digital Radios. Zigbee Is Based On An IEEE 802.15 Standard. Though Low Powered, Zigbee Devices Can Transmit Data Over Long Distances By Passing Data Through Intermediate Devices To Reach More Distant Ones, Creating A Mesh Network; I.E., A Network With No Centralized Control Or High-Power Transmitter/Receiver Able To Reach All Of The Networked Devices. The Decentralized Nature Of Such Wireless Ad Hoc Networks Makes Them Suitable For Applications Where A Central Node Can't Be Relied Upon. 


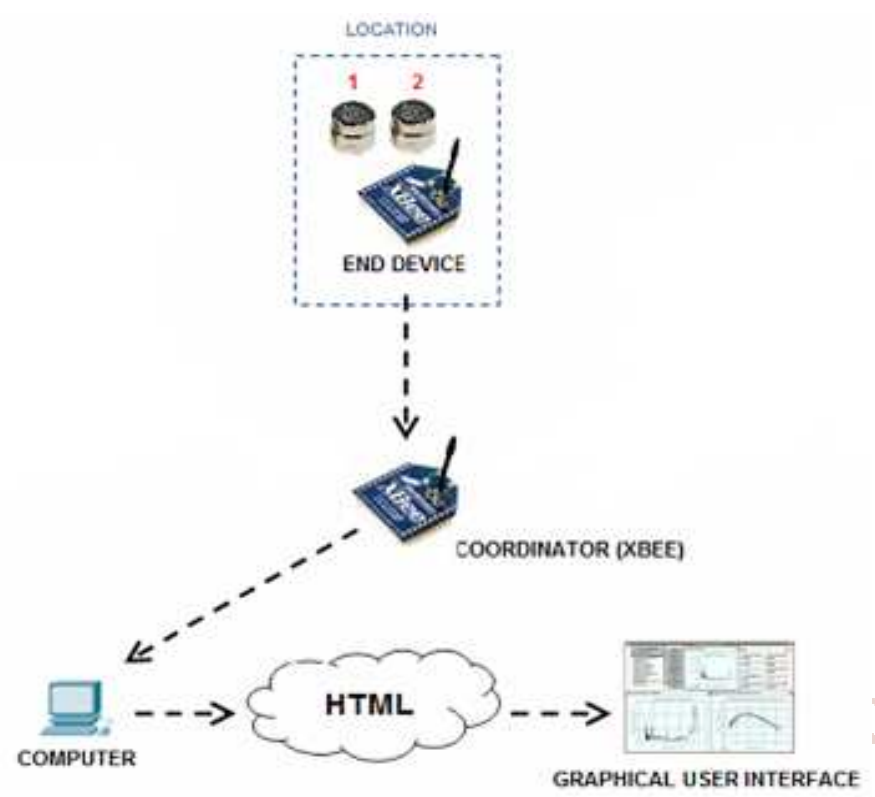

Figure1. Overall System

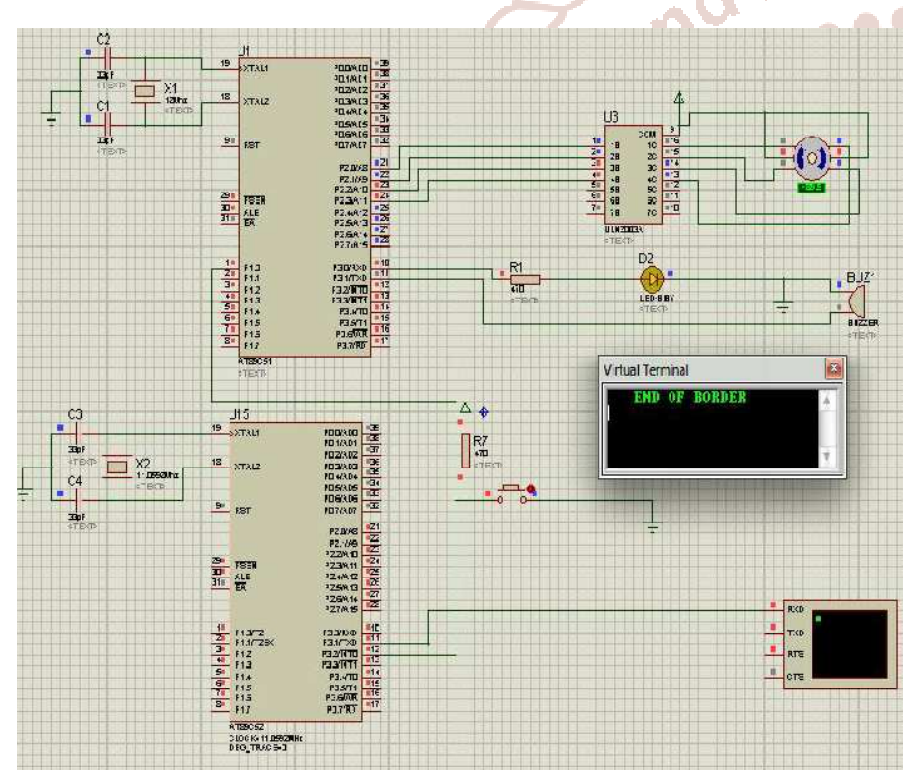

Figure2. System Hardware At Transmitter Side

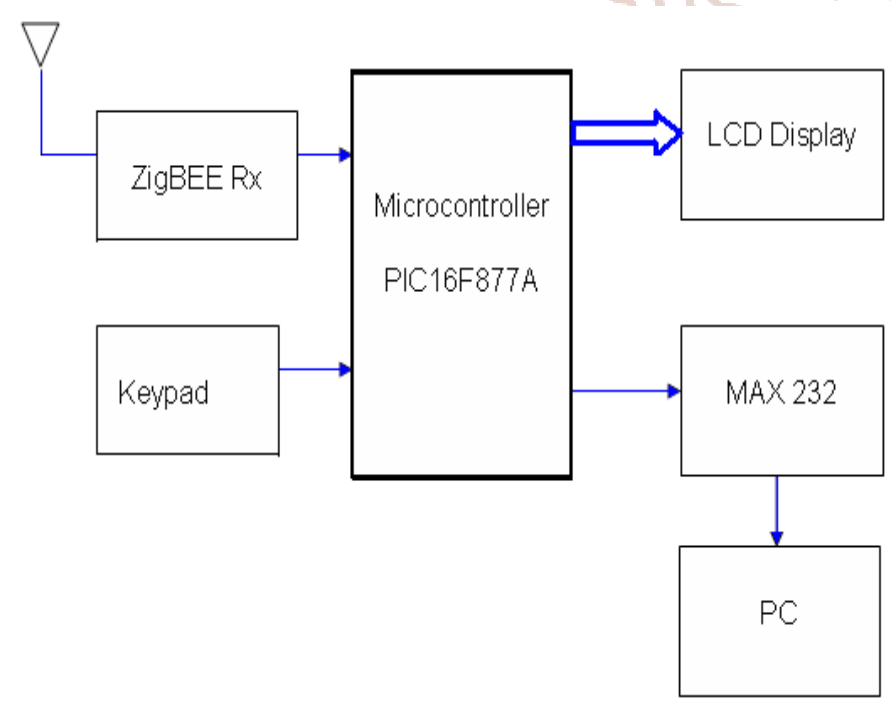

Figure3. System Hardware At Receiver Side

\section{HARDWARE DESCRIPTION}

The Sensor Platform Supports Data Collection, Storage and Offline Data Retrieval, They May Also Be Used For The Streaming Of Live Sensor Readings. System Can Be Accessed Through A USB Cable. This Wired Interface Provides Power To The Board. And This Power Can Be Used For On-Line Operation. Location and Time Information Is Provided By an On-Board GPS Module At $1 \mathrm{~Hz}$ Sampling Rate. Pollutant Levels Are Measured by Two Ana log Sensors: Viz LM35 and TGS 813. These Readings Along With GPS Information Are Stored In A Serial Flash Device 2MB. A 16X2 Character LCD Panel Provides Immediate Visualization Of Level Of Pollutant; GPS Readings.PIC 16F877 Microcontroller Controls Every Aspect Of The System From Battery Charging To Ana log To Digital Conversions. A Single chip Microcontroller PIC 16F877 Is Device That Has Built-In Resources Like Digital Input/output Ports, 16 Channels, 8/10 Bits Ana log-To-Digital Converter, 8 Input/output Interrupt-Driven Timers, RAM, EEPROM, FEEPROM Memory, RS-232 Serial Communication Ports, And SPI Communication Ports The Data Packet From The GPS-Module Includes An RMS Header Followed By UTC Time, Latitude, Longitude.. The GPS Modem Is Interfaced With The Microcontroller Using The RS-232 Communication Standard. The Central-Server Is Personal Computer With Accessibility To The Internet. The Pollution Server Is Connected To The Zigbee-Modem Via RS232 Communication Standard. The Air Pollution Information Is Saved To Database Of Central Server. Each Of These Components Is Described In The Following.

\section{A. PIC16F877A Microcontroller}

The PIC16F877A Microcontroller Is The Main Component of A Pollution Detection Unit. The Operating System That Runs Inside The Chip Coordinates The Substances Measurement Process, The Acquisition Of The GPS Coordinates And The Data Transmission To The Central Server. The Microcontroller Is Mounted On A Development Board That Provides An RS232 Serial Communication To The Zigbee Modem And GPS Receiver And A Parallel Connection To The Gas Sensors. The Connection Between The Gas Sensors And The PIC16F877A Microcontroller Can't Be Made Directly Because Of The Very Small Output Voltages Provided By The Sensors (Ma).This Problem Is Solved By Using Auxiliary Electronic Circuits For Signal Conversion Like OA (Operational Amplifiers) And Transistors. 


\section{B. Sensors Array}

The Sensor Array Consists Of Two Air Pollutions Sensors. Each Of The Sensors Has A Linear Current Output In The Range Of 4 Ma-20 Ma. The $4 \mathrm{Ma}$ Output Corresponds To Zero-Level Gas And The 20 Ma Corresponds To The Maximum Gas Level. A Simple Signal Conditioning Circuit Is Designed to Convert the 4 Ma-20 Ma Range Into $0-5 \mathrm{~V}$ to Be Compatible with the Voltage Range of The Built-In Ana log-To-Digital Converter in The PIC Microcontroller.

\section{GPS Reciever}

The GPS Module Provides The Physical Coordinate Location Of The Mobile-DAQ, Time And Date In National Marine Electronics Association (NMEA) Format. NEMA Format Includes The Complete Position, Velocity, And Time Computed By A GPS Receiver Where The Position Is Given In Latitude And Longitude. The Data Packet From The GPSModule Includes An RMS Header Followed By UTC Time, Data Validity Checksum, Latitude, Longitude, Velocity, Heading, Date, Magnetic Variation And Direction, Mode, And Checksum. The Only Information Required For The Proposed System Is Date, Time, Latitude And Longitude. The GPS Modem Is Interfaced With The Microcontroller Using The RS-232 Communication Standard.

\section{Zigbee Modules}

In This Paper, Two Types Zigbee Modules Are Used To Organize A Network For Air Pollution Monitoring System. The Network Is Controlled By Devices Called The Zigbee Coordinator Modem (ZCM). The Zcms Are Responsible For Collecting Data And Maintaining The Other Devices On The Network, And All Other Devices, Known As Zigbee End Devices (ZED), Directly Communicate With The ZCM. The Zigbee Module Is Hardware Platform Of Wireless Device.

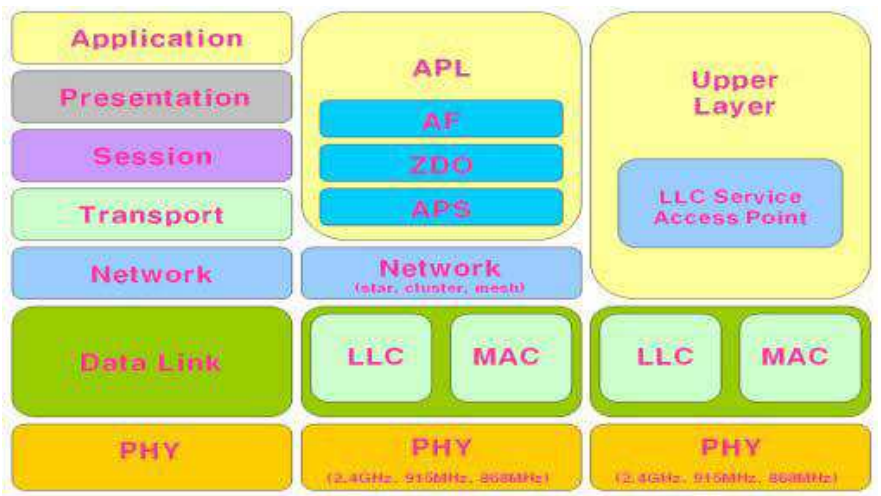

Figure4. Protocols Stack For Layered Wireless Communication
The Modules Realize The Basic Function Of Physical And MAC Layer, Such As Transmit And Receive, Modulation And Demodulation, Channel And Power Control.

RX

Frequency Band Coverage Data rate Ch Sensitivity Modulation $2.4 \mathrm{GHz}$ ISM Worldwide $250 \mathrm{Kbps} \quad 16 \quad-85 \mathrm{dbm} \quad 0$-QPSK $868 \mathrm{MHz}$ Europe $20 \mathrm{Kbps} \quad 1 \quad-92 \mathrm{dbm} \quad$ BPSK $915 \mathrm{MHz}$ ISM Americas $40 \mathrm{Kbps} 10-92 \mathrm{dbm}$ BPSK Figure5. Freguency Bands For Zigbee

Zigbee Operate At $2.4 \mathrm{GHz}$ Frequency ISM Band Wireless Communication. The Modules Include A Digital Direct Sequence Spread Spectrum Base Band Modem and an Effective Data Rate Of 250 Kbps. They Employ the EM2420 $2.4 \mathrm{GHz}$ Radio Frequency Transceiver and the ATMEL 8-Bit AVR Microcontroller. They Also Exhibit A Nominal Transmit Of $-1.5 \mathrm{dbm}$ And A Receive Sensitivity Of $92 \mathrm{dbm}$ When Powered At 3.0V, The Modules Draw 31.0ma In Transmit Mode And 28ma In Receive Mode. When The Entire Module Is In Sleep Mode, The Current Draw Is Reduced To Approximately 10ua.

\section{E. Central Server}

The Central-Server Is An Off-The-Shelf Standard Personal Computer With Accessibility To The Internet. The Pollution-Server Is Connected To The Zigbee-Modem Via RS-232 Communication Standard. The Air Pollution Information Sent From Each ZED Are Collected To ZCM. And Then The Data Are Saved To Database Of Central Server. Figure 5 Block Diagram of Zigbee Module

Clients Such As The Municipality, Environmental Protection Agencies, Travel Agencies, Insurance Companies And Tourist Companies Can Connect To The Central-Server Through The Internet And Check The Real-Time Air Pollutants Level Using A Normal Browser On A Standard PC Or A Mobile Device.

IV. System Implementation and the Resuls

The Sensor Output Voltages Representing The Level Of Gas For Each Pollutant Were Converted To A Ppm Value For Each Gas And Simultaneously Displayed On The LCD Display Of The Hardware. 


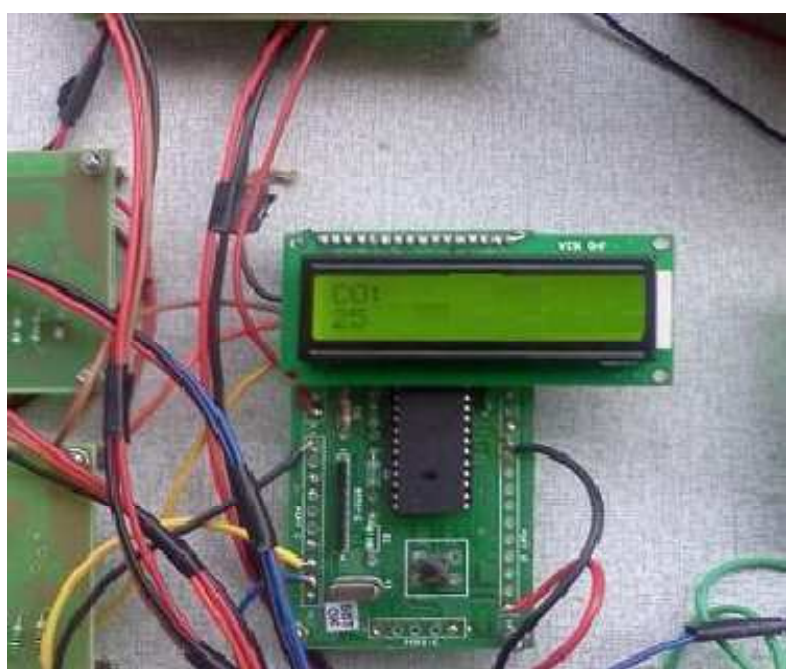

Figure6. Snapshot Of Pollutant Level On LCD

The Values of The Level Of Smoke And Temperature From The Serial Port Is Taken In Account By The Lab view Software Which Is Used To Display These Data In Their Respective Textbox And Also It Displayed In The Form Of Continuous Waveforms In Two Different Panel. The Screenshot of the Computer Window for the Lab view Designed Page Is Shown Below.

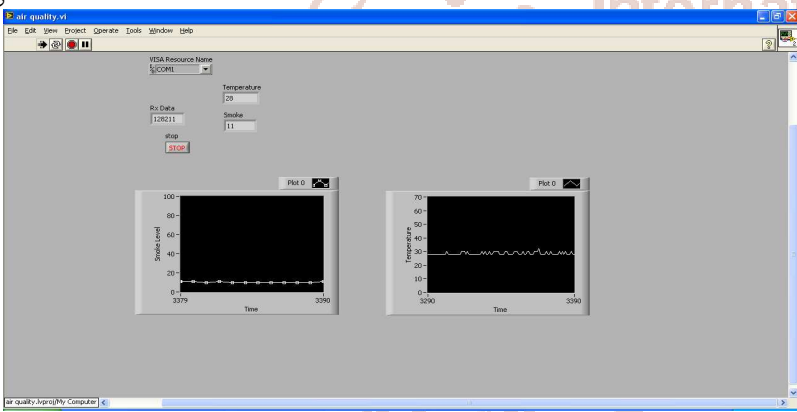

Figure7. Snapshot Of Output Window - After Start Sensing

The Air Pollution Monitoring System Includes a Feature Which Automatically Publishes the Results into Hyper Text Mark up Language (Html). The Figure 8 Shows The Interface Of The Website.

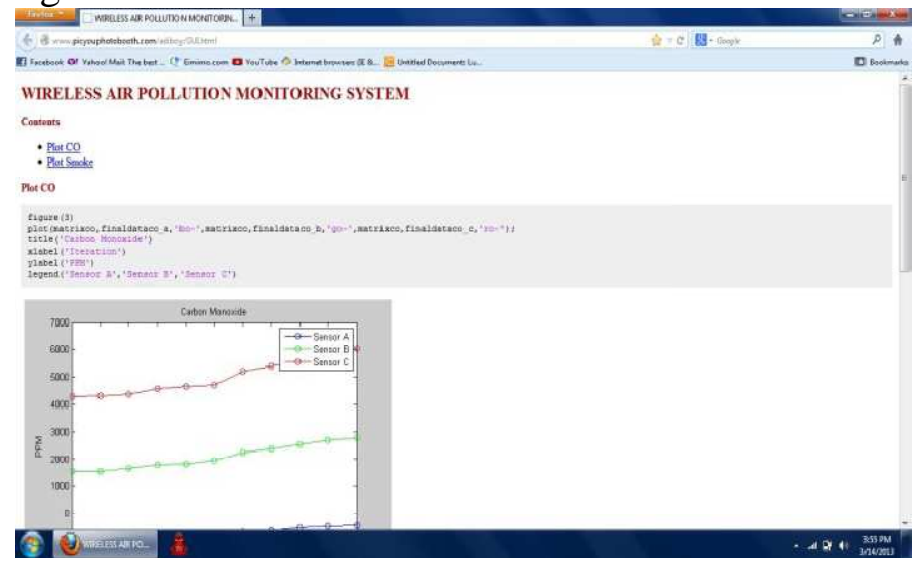

Figure8. Air Pollution Monitoring Web Interface

\section{CONCLUSION}

The System Detects Temperature And Smoke Emissions By Using Sensors Lm35 And Tgs 813 Which Is Commercially Available In The Local Market Makes It More Convenient And Variably Cheaper Compared To Pre-Ordered Sensors Abroad. This Sensor Saves 1/7 Of Power Consumption. All Data Were Treated Using A Developed Lab viewBased Program. The System's Overall Functionality Was Verified with Series of Temperature and Smoke Level Tests Including Tests in a Normal or Clean Environment, Cigarette Smoke, And Car Exhaust Emission on Different Time Intervals. The Cigarette Smoke Tests Showed That Carbon Monoxide Reading Is 6 Times Greater Than The Smoke Reading And For The Car Exhaust Tests It Showed The Carbon Monoxide Reading Is 4 Times Greater Than Smoke Reading. Systematized Data Logging Was Also Done To Back Up Every Result Which Will Be Of Valuable Reference During Erroneous Reading, Debugging And Calibration. The Results Were Processed Automatically Using A Lab view And My sql-Based Program With Output Published In Php Format Which Is Readily Available For Uploading In The Internet. The System Provided A Low Cost Data Acquisition Of Temperature And Smoke Pollutants That Can Be Read Online For Air Pollution Monitoring For Environmental Awareness.

\section{FUTURES COPE}

In Order To Make The Present Design More Realistic With Low Power By Replacing The Semiconductor Sensor, It Is Possible To Use Nano SENSOR; So That The Solar Power Base System Design Can Be Made Possible. For Longer Distance Communication We Can Use Gsm System Also We Can Control The Air Pollution By Using Sensed Data As Command.

\section{REFERENCES}

1. Alave, K., (2010). DENR Issues New Limits On Vehicular Emissions To Cut Air Pollution. Philippine Daily Inquirer, Pages 12-13.

2. Ageitos, M., Guerrero, P., Baldasano, J. (2008). Effects Of Introducing Hybrid Cars In Madrid And Barcelona Metropolitan Areas. Air Quality Management Strategies In Urban Areas, HARMO12-Conference, Pages 1-16.

3. Australianbureau Of Statistics. (2010). Environmental Awareness And Action. Australian Social Trends. 4102.0, Pages 1-5 
International Journal of Trend in Scientific Research and Development (IJTSRD) ISSN: 2456-6470

4. Bagula, A., Inggs, G., Scott, S., Zenarro, M. (2010). Community Sensor Networks:An Application To Pollution Maps. Wireless Communications And Information Conference, 2010.

5. Bashyal, S., Venayagamoorthy, G. (2007). Collaborative Routing Algorithm For Wireless Sensor Network Longevity. Real-Time Power And Intelligent System.ISSNIP1-4244-1502, Pages 515 $-521$

6. Burgonio, T. (2007). Air Pollution Kills Nearly 5,000 Metro Residents Yearly. Philippine Daily Inquirer, Pages 5 - 6Retrieved From Http://Newsinfo.Inquirer.Net

7. Choi, S., Kim, N., Cha, H., Ha, R., (2009). Micro Sensor Node For Air Pollutant Monitoring: Hardware And Software Issues. Sensors. ISSN 1424-82, Pages 7970 - 7987

8. Clean Air Initiative For Asian Cities (CAI-ASIA) Center. (2010). Air Quality Monitoring And Reporting. Air Quality In Asia: Status And Trends.2010 Edition, Page 13.

9. Congressional Commission on - Science Technology, And Engineering. (2010). Air Pollution Leading Cause Of Anemia, Infertility, Kidney Problems. Press Release - Angara.

10. Coyle, E., Bandyopadhyay, S. (2003). An Energy Efficient Hierarchical Clustering Algorithm For Wireless Sensor Networks. IEEE INFOCOM 2003, Pages 7803-7753

11. Denig. S. (2011). Assessing The Environmental Performance Of Asia's Major Cities. Asian Green City Index. Release No. A19100-F-P171-X-7600, Pages 4-63

12. Environmental Management Bureau Department Of Environment And Natural Resources. (2009). National Air Quality Status Report, 2005-2007, Pages 11 - 41. Retrieved From Http://Emb.Gov.Ph

13. Houk, J., Clagett M. (2009). Air Quality Management: Successes And Emerging Challenges.THE ENVIRONMENT AND TRANSPORTATION Making Both Better Than Before, TR NEWS 262, Pages 8-12.

14. Dr Invernizzi, G. (2004). Particulate Matter From Tobacco Versus Diesel Car Exhaust. Tobacco Control Unit, National Cancer Institute. Retrieved From:
Http://Www.Medicalnewstoday.Com/Releases/12 481.Php

15. Irudhayarj, A., Jaichandran, R., (2011). Prototype System For Monitoring And Computing Greenhouse Gases. World Of Computer Science And Information Technology Journal (WCSIT). Vol. 1, No. 5, Pages 177-183.

16. Khedo, K., Perseedoss, R., Mungur, A. (2010). A Wireless Sensor Network Air Pollution Monitoring System. International Journal of Wireless \& Mobile Network, Vol. 2, No. 2, Pages 1 - 15

17. Ma, Y., Richards, M., Ghanem, M., Guo, Y., Hassard, J. (2008). Air Pollution Monitoring And Mining Based On Sensor Grid In London. Sensors 2008, ISSN 1424-8220, Pages 3601 -3623

18. National Statistical Coordination Board. (2004). Pollutants From Different Sources, Metro Manila. Philippine Brown Environment Quality.AG-122005. Pages 1-2

19. Prasad, R., Baig, M., Mishra, R., Rajalakshmi, P., Desai, U., Merchant, S. (2011). Real Time Wireless Air Pollution Monitoring System. Next Generation Wireless Networks And Applications. Volume - 2, Issue - 2, Pages 370 - 375

20. Stankovic, J. (2006). Wireless Sensor Networks. Department Of Computer Science University Of Virginia Journal, Volume 10, Pages 5-25.

21. U.S. Environmental Protection Agency. (2009). Air Quality Index. A Guide to Air Quality and Your Health. EPA-456/F-09-002, Pages 3- 12

22. Xiang, Y., Bai, L., Piedrahita, R., Dick, R., Lu, Q., Hannigan, M., Shang, L. (2012). Collaborative Calibration and Sensor Placement for Mobile Sensor Networks. Computer-Communication Networks : Network Architecture And Design, IPSN'12, Pages 1 - 12 